\title{
MicroRNA-378d inhibits Glut4 by targeting Rsbn1 in vitamin $D$ deficient ovarian granulosa cells
}

\author{
HUITING SUN ${ }^{1,2}$, YICHAO SHI ${ }^{2}$, YUWEI SHANG ${ }^{2}$, XIA CHEN $^{2}$ and FEI XIA ${ }^{1}$ \\ ${ }^{1}$ Department of Reproductive Center, The First Affiliated Hospital of Soochow University, Suzhou, \\ Jiangsu 215006; ${ }^{2}$ Department of Reproductive Center, The Affiliated Changzhou No. 2 People's Hospital \\ of Nanjing Medical University, Changzhou, Jiangsu 213000, P.R. China
}

Received June 30, 2020; Accepted February 1, 2021

DOI: $10.3892 / \mathrm{mmr} .2021 .12008$

\begin{abstract}
Vitamin D (VD) is not only associated with bone growth and development, but is also closely associated with numerous other pathological conditions. The present study aimed to investigate the effect of microRNA (miRNA/miR)-378d on ovarian granulosa cells by regulating the round spermatid basic protein $1(R s b n l)$ in the absence of VD. The abnormal expression of miRNAs in ovarian tissues of the VD-deficient mouse was analyzed using transcriptome sequencing. miR-378d, glucose transporter 4 (Glut4) and aromatase (Cyp19a) expression levels were examined via reverse transcription-quantitative (RT-q)PCR and western blotting. The expression levels of Rsbn1, Glut4 and Cyp19a were detected in transfected mouse ovarian granulosa cells. The targeting regulation between miR-378d and Rsbnl was verified using double reporter gene assay and functional rescue experiments. Among the 672 miRNAs that were differentially expressed, cluster analysis revealed that 17 were significantly upregulated and 16 were significantly downregulated. Moreover, miR-378d showed significant upregulation, which was further verified via RT-qPCR. It was identified that the protein expression level of Rsbnl was significantly downregulated. Furthermore, Glut4 mRNA expression was significantly decreased in the mimic group but markedly increased in the inhibitor group. By contrast, the mRNA expression levels of Rsbnl and Cyp19a did not demonstrate any significant difference. The western blotting results indicated that the protein expression levels of Rsbnl and Glut4 were decreased and increased, respectively, while Cyp19a did not show any significant change. In addition, the double reporter gene experiments confirmed that $R s b n 1$ was the target gene of miR-378d. Collectively, the present results demonstrated that miR-378d
\end{abstract}

Correspondence to: Dr Fei Xia, Department of Reproductive Center, The First Affiliated Hospital of Soochow University, 899 Pinghai Road, Suzhou, Jiangsu 215006, P.R. China

E-mail: feixia0426@163.com

Key words: microRNA-378d, vitamin D, round spermatid basic protein 1 , ovarian granulosa cells was abnormally overexpressed in the ovarian tissues of the VD-deficient mice, and that miR-378d could inhibit Glut4 production by targeting $R s b n 1$, which may lead to insulin resistance.

\section{Introduction}

Over recent years, numerous studies have suggested that vitamin D (VD) is not only associated with bone growth and development $(1,2)$ but that it is also closely associated with the cognitive functioning in psychotic disorders (3), autoimmune diseases (4), cardiovascular diseases (5) and metabolic diseases $(6,7)$. According to the data on standard VD requirements, VD insufficiency or deficiency is globally widespread, and is present in $50-80 \%$ of the total global population (8). Previous studies have reported that the VD receptor (VDR) is present in the human placenta (9), uterine decidua, pituitary (10) and ovarian granulosa cells $(11,12)$. VD can promote the production of estradiol, estrone, progesterone and insulin-like growth factor binding protein 1 in the ovary, and these hormones and growth factors have positive roles in the development of follicles and embryos (12). Moreover, VD deficiency can reduce endometrial receptivity, which can, in turn, affect reproductive functions (13). Jiang et al (14) have revealed that $1,25(\mathrm{OH}) 2 \mathrm{D} 3$ can upregulate the mRNA and protein expression levels of homeobox gene A10 (Hoxa10) by binding with endometrial stromal cell receptors. Hoxa10 is essential for female fertility and embryo implantation, and lack of VD could reduce the expression level of Hoxa10, thus affecting female fertility. Furthermore, Wei et al (15) have reported that VDR is a key regulator of inflammatory responses and $\beta$-cell survival.

Several studies have reported that microRNAs (miRNAs/miRs) are abundant in the female reproductive system, among which miR-26a and miR-125b are most abundantly expressed in the ovaries (16). miR-378d is a member of the miR-378 family, which has eight members (miR-378 $\mathrm{a} / \mathrm{b} / \mathrm{c} / \mathrm{d} / \mathrm{e} / \mathrm{f} / \mathrm{h} / \mathrm{i})$. As these share similar seed sequences, such as a sequence of 6-8 nucleotides that are critical for mRNA target recognition, they are considered to have similar activity and targets (17). Research has mainly focused on tumor, heart and liver diseases, where miR-378a is the most widely studied member, and miR-378d the much less studied factor (18-21). 
Ishida et al (22) reported that miR-378 was closely associated with adiponectin expression. Gungormez et al (23) revealed that the expression levels of miRNAs from the miR-378 family are decreased in the tumor tissues of patients with stage II colon cancer compared with the normal tissues, which could be used as a biomarker for early colon cancer. Moreover, miR-378d-2 has been reported to be closely associated with tumor generation in obese patients with breast cancer (24).

The present study aimed to examine miR-378d, Glut4 and aromatase (Cyp19a) expression levels in mouse ovarian tissues. GLUT4 is a member of the facilitative GLUT family, characterized by preferential expression in muscle and adipose tissue, and is responsible for insulin-stimulated glucose uptake (25). The CYP19 gene, which is widely known to encode aromatase, is the key enzyme for estrogen production (26).

The effect of VD on the reproductive system in female mice is achieved by the differential expression of miRNAs (27). In the present study, a mouse model of VD deficiency was established to investigate the molecular mechanisms underlying the effects of miRNAs on ovarian granulosa cells in female mice with VD deficiency.

\section{Materials and methods}

Animals. Female C57BL/6 J mice (age, 3 weeks; weight, 12-18 g) were obtained from the Carvens Animal Laboratory Technology Company [http://www.cavens.com.cn/index.php; animal certificate no. SCXK(SU)2016-0010]. All procedures related to animal use were approved by the Animal Care and Use Committee of Nanjing Medical University. This study was carried out in strict accordance with the recommendations in the Guide for the Care and Use of Laboratory Animals of the National Institutes of Health (28). Mice were housed in a specific pathogen-free environment under standard housing conditions with controlled temperature $\left(22 \pm 1^{\circ} \mathrm{C}\right)$ and humidity $(52 \pm 5 \%)$. Mice were exposed to a 12-h light/dark cycle and had ad libitum access to the assigned food and water. Mice were weighed weekly and monitored for changes in health status.

After acclimatization for 1 week, the mice were randomized to VD-deficient (25 IU VD3/kg; cat. no. Dyets D 119289) or control diet (5,000 IU VD3/kg; cat. no. Dyets D 119290) groups for 8 weeks. All diets were procured from Dyets, Inc.

In the present study a total of 16 mice were used, of which 5 were the control group and 6 were VD deficient group, and the remaining 5 were used to extract mouse ovarian granulosa cells. After 8 weeks, mice from each group were abdominally anesthetized with $4 \%$ chloral hydrate $(370-400 \mathrm{mg} / \mathrm{kg})$ and then bled from the retro-orbital venous plexus, after which they were sacrificed by cervical dislocation; efforts were made to minimize animal suffering (28). The blood (20-50 $\mu \mathrm{l}$ per mouse) was centrifuged at $1,000 \mathrm{x}$ g for $10 \mathrm{~min}$ at room temperature, and the serum was collected for 25-(OH) D3 analysis. Serum 25-(OH) D3 levels were measured using an ELISA kit (cat. no. AC-57SF1; Immunodiagnostic Systems, Ltd.) to confirm VD deficiency. Ovarian tissues were collected for RNA sequencing, reverse transcription-quantitative (RT-q) PCR and western blot analysis.

In order to confirm whether miR-378d post-transcriptionally regulates $R s b n l$ in ovaries, normal mouse granulosa cells were isolated from ovarian follicles and cultured. The granulosa cells were cultured in DMEM/F-12 (1:1; cat. no. 11330-057) supplemented with $10 \%$ fetal bovine serum (cat. no. 10270) and $100 \mathrm{U} / \mathrm{ml}$ penicillin plus $100 \mu \mathrm{g} / \mathrm{ml}$ streptomycin (cat. no. 15140; all Thermo Fisher Scientific, Inc.) at $37^{\circ} \mathrm{C}$ with $5 \% \mathrm{CO}_{2}$. The miR-378d mimic (5'-ACUGGCCUUGGAGUCAGAAGGU-3'), inhibitor (5'-ACCUUCUGACUCCAAGGCCAGU-3') and their control sequences were synthesized by Shanghai GenePharma Co., Ltd. Once cells reached $80 \%$ confluency, they were digested by $0.25 \%$ trypsin (Thermo Fisher Scientific, Inc.) at room temperature for $2 \mathrm{~min}$, counted by Countess 3 Automated Cell Counters (Thermo Fisher Scientific, Inc.) and seeded into 6-well plates in growth medium without antibiotics at a density of $2.0 \times 10^{5}$ cells/well. After culturing at $37^{\circ} \mathrm{C}$ for 18-24 h when $70 \%$ confluency was reached, cells were transfected with $50 \mathrm{nM}$ miR-378d mimic or miR-378d inhibitor using Lipofectamine ${ }^{\circledR}$ RNAi MAX reagent (Invitrogen; Thermo Fisher Scientific, Inc.), according to the manufacturer's protocol. At $48 \mathrm{~h}$ post transfection, cells were harvested for RNA isolation or protein extraction. Transfection efficiency was measured by RT-qPCR for miR-378d. All transfection experiments were performed in duplicate wells and repeated three times before protein and mRNA expression levels of Rsbn1, Glut4 and Cyp19a were assessed.

RNA sequencing. The total RNA of each sample was extracted using TRIzol ${ }^{\circledR}$ reagent (Invitrogen; Thermo Fisher Scientific, Inc.) and an miRNeasy Mini kit (Qiagen, Inc.), and was quantified using an Agilent 2100 Bioanalyzer (Agilent Technologies, Inc.), NanoDrop system (Thermo Fisher Scientific Inc.) and $1 \%$ agarose gel. A total of $2 \mu \mathrm{g}$ total RNA with RNA integrity number $>7.5$ was used for subsequent library preparation. Next-generation sequencing library preparations were constructed according to the manufacturer's protocol (NEBNext ${ }^{\circledR}$ Multiplex Small RNA Library Prep Set for Illumina ${ }^{\circledR}$; Illumina, Inc.). The 3' SR Adaptor for Illumina was ligated to the small RNA using 3'Ligation Enzyme. To prevent adaptor-dimer, the excess of 3' SR Adaptor was hybrid with SR RT Primer for Illumina. The 5' SR Adaptor for Illumina was ligated to the small RNA using 5'Ligation Enzyme, and the first-strand cDNA was synthesized using ProtoScript II Reverse Transcriptase (New England Biolabs). Each sample was then amplified by PCR for 12 cycles using P5 (GTTCAGAGTTCTACAGTCCGACGATCNNNN) and P7 (NNNNTGGAATTCTCGGGTGCCAAGG; both NextFlex) primers, with both primers carrying sequences that can anneal with the flow cell to perform bridge PCR, and the P7 primer carrying a six-base index allowed for multiplexing. PCR was performed under the following conditions: Initial denaturation at $94^{\circ} \mathrm{C}$ for $30 \mathrm{sec}$, followed by 12 cycles of $94^{\circ} \mathrm{C}$ for $15 \mathrm{sec}$, $62^{\circ} \mathrm{C}$ for $30 \mathrm{sec}$ and $70^{\circ} \mathrm{C}$ for $15 \mathrm{sec}$ and final extension at $70^{\circ} \mathrm{C}$ for $5 \mathrm{~min}$.

RT was performed according to the instructions of the Vazyme RT kit (cat. no. R223-01; Vazyme Biotech Co., Ltd.). The reactants (volume, $10 \mu \mathrm{l}$ ) were added into $1.5 \mathrm{ml}$ centrifuge tube in the following order: $5 \mathrm{X}$ HiScript II Select qRT SuperMix $(2 \mu \mathrm{l})$, Oligo(dT) $(1 \mu \mathrm{l})$, TotalRNA $(1 \mu \mathrm{l})$ and RNase Free $\mathrm{H}_{2} \mathrm{O}$ (add water to $10 \mu \mathrm{l})$. PCR reaction condition were as follows: Initial denaturation at $95^{\circ} \mathrm{C}$ for $30 \mathrm{sec}$, followed by 40 cycles at $95^{\circ} \mathrm{C}$ for $10 \mathrm{sec}, 60^{\circ} \mathrm{C}$ for $20 \mathrm{sec}$ and $72^{\circ} \mathrm{C}$ for $1 \mathrm{~min}$. The RT primer 
Table I. List of forward and reverse primers.

\begin{tabular}{lcc}
\hline Gene & Forward primer & Reverse primer \\
\hline GAPDH & 5'-AACGACCCCTTCATTGAC-3' & 5'-TCCACGACATACTCAGCAC-3' \\
Rsbn1 & 5'-AGGTCTCAGAGCGATGACGG-3' & 5'-GGGTTCACTTGTCCGAGGTAG-3' \\
Cyplisa & 5'-TGGCCTTCTTTGAGATTGGC-3' & 5'-AACCCATGCCGACAATGAAG-3' \\
U6 & 5'-AACAACAACCCGAGCCTTTG-3' & 5'-AATGCTGCTTGATGGACTCC-3' \\
& 5'-CTCGCTTCGGCAGCACA-3' & 5'-AACGCTTCACGAATTTGCGT-3'
\end{tabular}

Glut4, glucose transporter 4; Cyp19a, aromatase; Rsbn1, round spermatid basic protein 1 .

sequence was 5'-GTCGTATCCAGTGCAGGGTCCGA GGTATTCGCACTGGATACGACACCTTC-3'. Further details of the primers are provided in Table I.

The PCR products of $\sim 140$ bp were validated using an Agilent 2100 Bioanalyzer (Agilent Technologies, Inc.), and quantified using a Qubit 2.0 Fluorometer (Invitrogen; Thermo Fisher Scientific, Inc.). Then, libraries with different indexes were multiplexed and loaded on an Illumina HiSeq instrument according to the manufacturer's instructions (Illumina, Inc.). Sequencing was performed using a $1 \times 50$ single-end (SE)/2x150 paired-end configuration; image analysis and base calling were conducted using the HiSeq Control Software v2.2.58 + OLB + GAPipeline-1.6 (Illumina, Inc.) on the HiSeq instrument. The sequences were processed and analyzed by Genewiz, Inc. Libraries were sequenced, and ovaries tissue miRNA RNA sequences were generated with the Illumina HiSeq $\mathrm{X}$ sequencing platform by Cofactor Genomics, Inc.

Gene Ontology (GO)-TermFinder (https://metacpan. org/pod/GO::TermFinder) was used to identify GO terms that annotate a list of enriched genes with a significant $\mathrm{P}<0.05$. Kyoto Encyclopedia of Genes and Genomes (KEGG) is a collection of databases dealing with genomes, biological pathways, diseases, drugs and chemical substances (https://www. genome.jp/kegg). In-house scripts were used to enrich significant differentially expressed genes in KEGG pathways.

Prediction of target genes and luciferase reporter assays. Using miRNA target gene prediction programs TargetScan 7.2 (targetscan.org/vert_72/) and miRDB (mirdb. org/cgi-bin/search.cgi), the putative miR-378d binding sites at the 3'untranslated region (UTR) of potential miR-378d target genes in mouse were analyzed. Based on the seed region sequences of the candidate target genes provided by the National Center for Biotechnology Information, primers targeting the gene segments containing the seed sequences were designed with SpeI-HindIII restriction enzyme sites incorporated. In addition, mutagenic primers were designed to specifically mutate $3-5$ bases in the seed region of the candidate target genes. The primer sequences are listed in Table I. After primer annealing, candidate target sequences containing wild-type (WT) and mutant (MUT) seed regions were synthesized. The WT and MUT sequences were cloned into pMIR-Report Luciferase [General Biosystems (Anhui) Co. Ltd.] to construct WT and MUT reporter plasmids, respectively.
The WT and MUT sequences of the 3'UTR of round spermatid basic protein 1 (RSBN1) were developed and named $R S B N 1-3$ 'UTR WT and RSBN1-3'UTR MUT. Next, $100 \mathrm{ng} R S B N 1-3$ 'UTR WT or RSBN1-3'UTR MUT and $0.25 \mu \mathrm{l}$ miR-378d mimic and inhibitor $(20 \mu \mathrm{M})$, or suitable negative controls (NC) were mixed with LipoPlus (B. Braun Medical Indonesia) following the manufacturer's instruction. Following the plasmid mixture into the transfected media, the plasmid was co-transfected into the $\mathrm{C} 57 \mathrm{~B} / \mathrm{L} 6$ mouse granulosa cell line. The cells were cultured at $37^{\circ} \mathrm{C}$ with $5 \% \mathrm{CO}_{2}$ for $48 \mathrm{~h}$. When the cells reached $70-80 \%$ confluence, they were harvested for further analysis. After the cells were harvested, Renilla luciferase and firefly luciferase activities were measured using a luciferase assay kit (Thermo Fisher Scientific, Inc.) and a chemiluminescence reader (FL600 Fluorescence Microplate Reader; BioTek Instruments, Inc).

In order to evaluate the ability of miR-378d binding to the 3'UTR of RSBN1 in mouse, a luciferase reporter assay was conducted in mouse granulosa cells. Female C57BL/6 mice (age, 21 days; weight, 12-18 g) were injected intraperitoneally (i.p.) with 5 IU pregnant mare serum gonadotropin (PMSG; Prospec-Tany TechnoGene, Ltd.) to initiate follicular development. Mice were then euthanized by cervical dislocation $48 \mathrm{~h}$ after PMSG injection, then the ovaries were removed. Granulosa cells were obtained from the ovarian tissue via follicular puncture and placed in PBS. The cell suspension was filtered through a $70-\mu \mathrm{m}$ mesh sieve three times to remove oocytes and centrifuged at $1,000 \mathrm{x} \mathrm{g}$ for $5 \mathrm{~min}$ at room temperature. The pellet was re-suspended in serum-free DMEM F12 insulin-transferrin-sodium selenite (Sigma-Aldrich; Merck KGaA).

PMSG injection to initiate follicular development. Next, the miR-378d binding capability to the 3'UTR of RSBN1 was examined in mice, and a luciferase reporter assay was conducted in mouse granulosa cells. Female C57BL/6 mice (age, 21 days; weight, 12-18 g) were injected i.p. with 5 IU PMSG (Prospec-Tany, TechnoGene, Ltd.) to initiate follicular development. Next, the mice were sacrificed by cervical dislocation $48 \mathrm{~h}$ later after PMSG injection, after which the ovaries were removed, using a $1 \mathrm{ml}$ syringe with a needle. The PMSG injection was performed by the method as described previously (29).

Rescue experiments. To construct Rsbnl overexpression plasmid, RSBN1 forward and reverse (RSBN1 forward, 
Table II. Primers targeting the seed region of the candidate target genes.

\begin{tabular}{ll}
\hline Gene & Sequence \\
\hline 3'UTR sequence of $R s b n 1$ & 5'-ACTAGTAGCAGATGCCATCCTGTCATCTAAGCTGGTCATTACTAA \\
& TACACAAGGAGACTGTCTCCTGACAGCCAGCACTGTGCAATCA \\
Mutation sequence of $R s b n l$ & CTCAGGAACCAGCGGATCTGCAAAGACCAAGCTT-3' \\
& 5'-ACTAGTAGCAGATGCCATCCTGTCATCTAAGCTGGTCATTACTA \\
& ATACACAAGGAGAGTCTGTGCTGACACCGACCACTGTGCAAT \\
& CACTCAGGAACCAGCGGATCTGCAAAGACCAAGCTT-3'
\end{tabular}

Rsbn1, round spermatid basic protein 1; UTR, untranslated region.

5'-TAAGCTTGGatgttcatctctggacga-3' and reverse, 5'-TGGAT CCGAGtaaaggacaaatcagact-3') primers were used to amplify the RSBN1 sequence. The amplified product was digested by HindIII and NotI enzymes and connected with pcDNA3.1 (+) digested by the same enzymes to form a recombinant plasmid. The recombinant plasmid was transformed into $E$. coli to obtain stable Rsbnl overexpression plasmid strain.

For the rescue experiments, mouse granulosa cells were first transfected using Lipofectamine ${ }^{\mathrm{TM}} 2000$ transfection reagent (Invitrogen; Thermo Fisher Scientific, Inc.) according to the manufacturer's instructions with $R s b n 1$ small interfering (si)RNA(5'-GCAUACAUGGACGAACUCUTT-3', Shanghai GenePharma Co., Ltd.), negative control (NC; 5'-GTCCAGAA CGAATTTATAAGT-3', Shanghai GenePharma Co., Ltd.), miR-378d mimic, miR-378d inhibitor (50 pmol/ml)or Rsbnl $\mathrm{OE}(1.67 \mu \mathrm{g} / \mathrm{ml})$ at $37^{\circ} \mathrm{C}$ for $4 \mathrm{~h}$. The following groups were established in the $\mathrm{C} 57 \mathrm{~B} / \mathrm{L} 6$ mouse granulosa cells: $\mathrm{NC}+$ control vector, miR378d mimic + control vector, $\mathrm{NC}+$ Rsbnl OE, miR378d mimic + Rsbnl OE, inhibitor $\mathrm{NC}+$ siRNA NC, miR-378d inhibitor + siRNA NC, inhibitor $\mathrm{NC}+R s b n 1$ KD, miR-378d inhibitor $+R s b n 1$ KD. After another $48 \mathrm{~h}$, cells were harvested for qPCR and western blot analysis.

$R T$ - $q P C R$. Total RNA was isolated from ovary tissue or cultured cells and extracted using TRIzol ${ }^{\circledR}$ reagent (Invitrogen; Thermo Fisher Scientific, Inc.) according to the manufacturer's instructions. The concentration of total RNA was measured by Ultraviolet Spectrophotometer at $260 \mathrm{~nm}$ (Thermo Fisher Scientific, Inc.). cDNA was synthesized from $1 \mu \mathrm{g}$ total RNA using an All-in-one TM miRNA qPCR Detection kit (GeneCopoeia, Inc.) or from 500 ng total RNA using a HiScript II RT SuperMix according to the manufacturer's instructions (Vazyme Biotech Co., Ltd.). qPCR analysis was performed using AceQ qPCR SYBR Green master mix (Vazyme Biotech Co., Ltd.) on an ABI ViiA 7 Real-Time PCR system (ABI, USA). The qPCR was performed under the following conditions: Initial denaturation at $95^{\circ} \mathrm{C}$ for $30 \mathrm{sec}$, followed by 40 cycles of $95^{\circ} \mathrm{C}$ for $10 \mathrm{sec}, 60^{\circ} \mathrm{C}$ for $20 \mathrm{sec}$ and $72^{\circ} \mathrm{C}$ for $1 \mathrm{~min}$. U6 snRNA was used as an internal control for miR-378d and GAPDH was used as an internal control for other genes. Experimental data were analyzed using a relative quantification method $\left(2^{-\Delta \Delta C q}\right)$ (30). All experiments were repeated three times. The RT-qPCR primers are described in detail in Table II.
Western blotting. The mouse ovary tissue and granulosa cells were rinsed with PBS, treated with $0.25 \%$ trypsin (Gibco; Thermo Fisher Scientific, Inc.) for $2 \mathrm{~min}$ at $37^{\circ} \mathrm{C}$, harvested and transferred to centrifuge tubes. Cellular proteins were extracted using the RIPA lysis buffer (cat. no. R0020; Beijing Solarbio Science \& Technology Co., Ltd.) and the protein concentration was measured using a BCA kit (cat. no. P0012S; Beyotime Institute of Biotechnology), after which $50 \mu \mathrm{g}$ protein samples were separated via SDS-PAGE on a $12 \%$ gel and transferred to a PVDF membrane (cat. no. 3010040001; Sigma-Aldrich; Merck KGaA). The membrane was blocked for $2 \mathrm{~h}$ with blocking buffer (cat. no. P0023B; Beyotime Institute of Biotechnology) at room temperature and then incubated with the following primary antibodies (all 1:1,000) diluted with primary antibody dilution buffer (cat. no. P0023A; Beyotime Institute of Biotechnology): Anti-Rsbnl rabbit polyclonal IgG (cat. no. NBP1-57724; Novus Biologicals, LLC), Ras-related nuclear-binding protein 10 (RANBP10) rabbit polyclonal IgG (cat. no. ab172730; Abcam) or GAPDH mouse monoclonal IgG1 (cat. no. G8795; Sigma-Aldrich; Merck KGaA) for $12 \mathrm{~h}$ at $4^{\circ} \mathrm{C}$ on a shaker. The membrane was then incubated with corresponding secondary antibodies (goat anti-rabbit IgG-HRP, cat. no. AP307P, Sigma-Aldrich; Merck KGaA; or goat anti-mouse IgG-HRP, cat. no. ab97023, Abcam; 1:5,000) at room temperature for $1 \mathrm{~h}$. The protein was detected by chemiluminescence using Pierce ECL Western Blotting Substrate (Thermo Fisher Scientific, Inc.). The optical density of protein fragments was quantified by ImageJ (version 1.25p; National Institutes of Health).

Statistical analysis. GraphPad Prism 5.01 (GraphPad Software, Inc.) and ImageJ (National Institutes of Health) were used for raw data analysis. Data are presented as the means \pm SD $(n=3)$. The differences between two groups were analyzed using unpaired Student's t-test. For multiple groups, one-way ANOVA followed by Bonferroni test was used. $\mathrm{P}<0.05$ was considered to indicate a statistically significant difference.

\section{Results}

Mouse model of VD deficiency. C57BL/6J mice that were nutritionally deficient in VD were established as previously described (27). After 8 weeks on a diet, VD-mice $(n=6)$ had 25-(OH) D3 levels of $5.99 \pm 2.30 \mathrm{ng} / \mathrm{ml}$ compared with control mice $(\mathrm{n}=5)$ that had 25-(OH) D3 levels of $46.70 \pm 10.17 \mathrm{ng} / \mathrm{ml}(\mathrm{P}<0.0001$; 
A

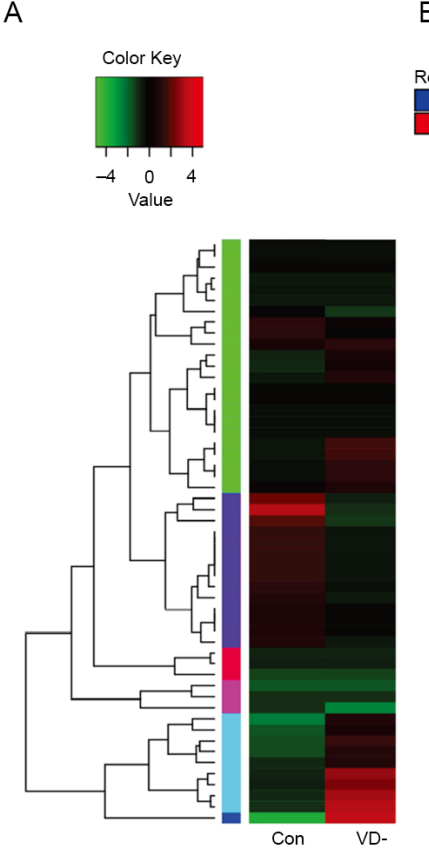

B

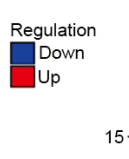

C

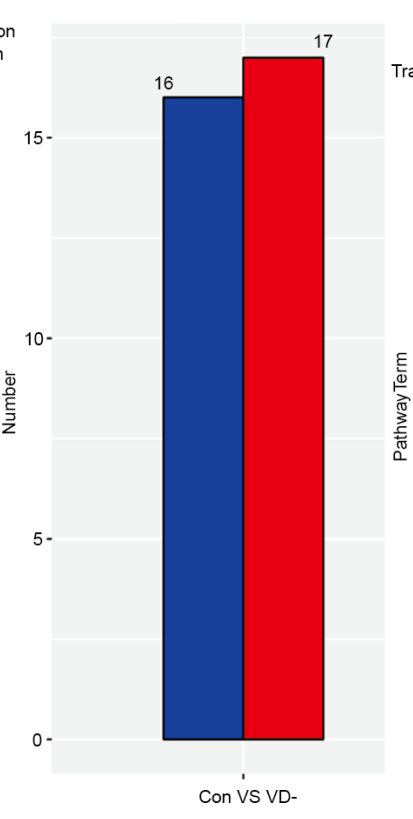

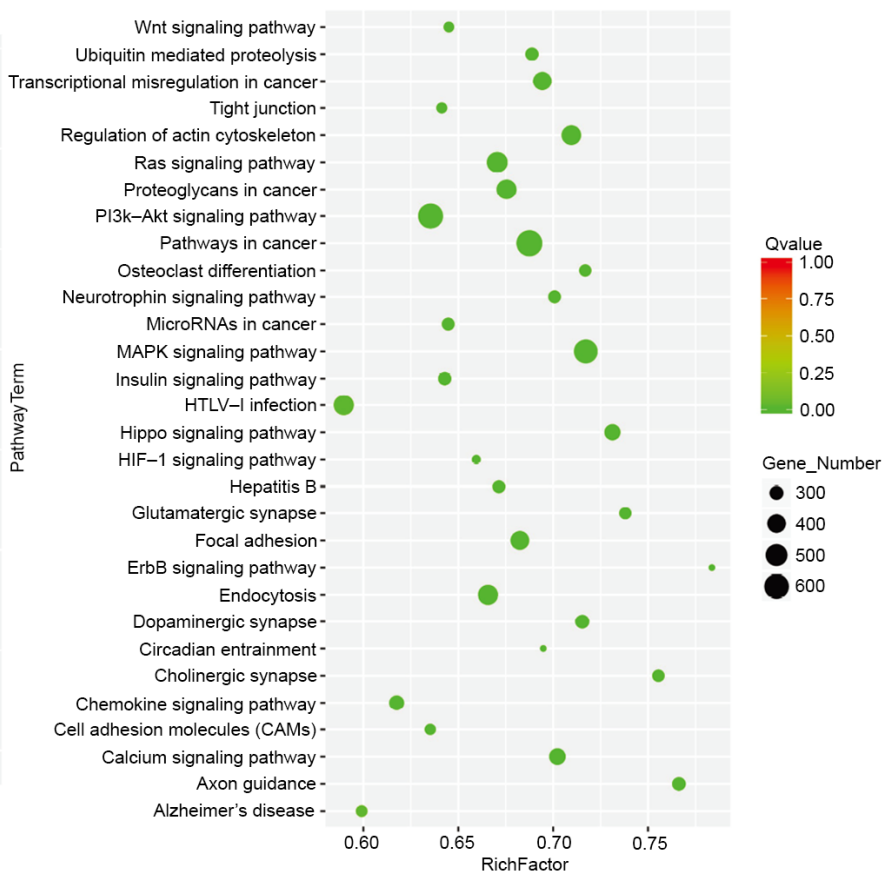

Figure 1. Transcriptome sequencing analysis of ovary tissues from VD- and control mice. (A) miRNA clustering analysis of VD deficient mice using a heat map to show the significantly downregulated gene number. Differential miRNA clustering diagram. The $\log 2$ TPM value was used for clustering. Red indicated highly expressed miRNAs, while green indicated lowly expressed miRNAs. (B) DESeq test results were screened according to the significant difference criteria (differential miRNA expression changes were $>2$ times and false discovery rate $\leq 0.05$ ), and the significant downregulation of miRNA expression was measured. (C) Scatter plot of KEGG enrichment of differential miRNA target genes. VD, vitamin D; miRNA, microRNA; Con, control.

Table SI). No significant changes were observed in serum calcium and phosphorus concentrations at 8 weeks on VD-deficient or control diet (data not shown). The average weight was similar in mice on VD- and control chow over the 8 weeks (Table SI).

Functional enrichment analysis and transcriptional signature analysis of ovary tissues from VD- and control mice. RNA sequencing was conducted using total RNA isolated from ovary tissues of control and VD-mice at the age of 11 weeks. A total of 672 differentially expressed miRNAs were identified, while transcripts per million (TPM) values of $>4$ in both libraries were retained for further analysis (Fig. 1A). Among them, 33 miRNAs were reported having $\log 2$-fold change and false discovery rate $\leq 0.05$ ) in upregulation or downregulation, regardless of miRNA abundance (Table SII). Furthermore, 17 miRNAs were significantly upregulated, while 16 miRNAs were downregulated in the ovarian tissue of VD-mice compared with the control (Fig. 1B).

KEGG enrichment analysis was used to analyze the significant biological processes. Most of the target genes were associated with 'cell binding' and 'catalytic activity' and were involved in multiple functions such as 'biological regulation' and 'molecular process of organelles'. Numerous essential biological processes were changed, such as 'ubiquitin-mediated proteolysis', 'cell cycle', 'Wnt signaling pathway', 'calcium signaling pathway', 'MAPK signaling pathway' and 'hypoxia inducible factor 1 (HIF-1) signaling pathway', amongst others (Fig. 1C).

Expression of miR-378d in mouse ovarian tissue as determined via RT-qPCR. RNA-seq has demonstrated miR-378d was significantly upregulated in the ovaries of VD mouse models (data not shown). Importantly, a recent study reported that hsa-miR-378d may be a novel and potential biomarker for improving the diagnosis of colorectal cancer (31).

miR-378d expression in VD-mouse ovarian tissue was detected via qPCR and compared with the control group. The miR-378d expression was significantly higher compared with that in the control group $(\mathrm{P}<0.001$; Fig. 2A). Bioinformatic analysis using the two online prediction algorithms that were previously mentioned was first conducted to predict the genes targeted by miR-378d. As a result, a total of 11 potential target genes were identified, including methyl-CpG binding protein 2, neurofibromin 1, BICD cargo adaptor 2, ETS proto-oncogene 1, growth arrest specific 7 , GTP binding protein 2, LIM domain containing 1, polycomb group ring finger 3, RSBN1, SIM bHLH transcription factor 1 and tetraspanin 9. The bioinformatic analysis demonstrated that there is a high complementary sequence between Rsbn110 3'UTR and miR-378d. Rsbnl protein expression level was significantly downregulated in the VD-deficiency group compared with the control group ( $\mathrm{P}<0.001$; Fig. $2 \mathrm{C}$ and $\mathrm{D})$, while Rsbnl mRNA expression level was not significantly altered (Fig. 2B).

miR-378d regulates RSBN1 in mouse granulosa cells. RT-qPCR results identified that the miR-378d expression levels were significantly upregulated in the miR-378d mimics group compared with the $\mathrm{NC}$ group $(\mathrm{P}<0.001)$. Moreover, the miR-378d expression level was significantly downregulated in the miR-378d inhibitor group compared with the inhibitor NC group $(\mathrm{P}<0.001)$. These results indicated the successful overexpression or knockdown of miR-378d in mouse granulosa cells (Fig. 3A). 
A

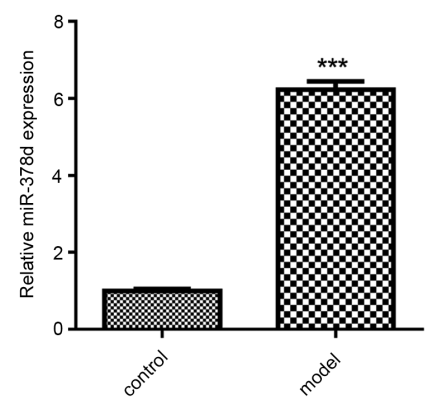

C

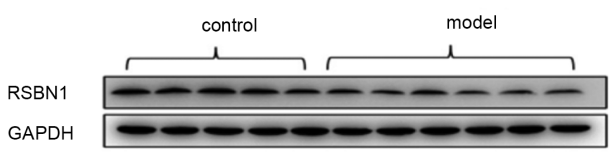

B
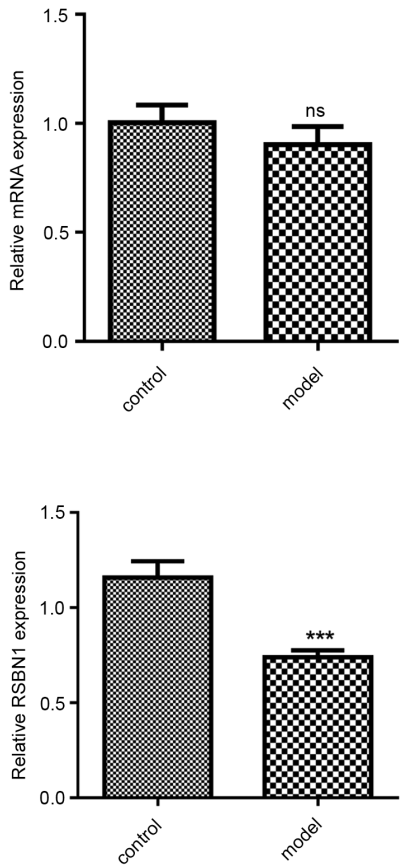

Figure 2. Expression levels of miR-378d and Rsbn1 in VD-deficient mouse ovaries. (A) miR-378d expression levels were significantly upregulated in the VD-deficiency group compared with the control group. (B) Relative expression of Rsbnl mRNA in mouse ovaries, compared with control group. (C) Western blot analyses of Rsbn1 and GAPDH expression levels in ovary tissue of VD-group and control group. (D) The optical density of Rsbn1 was quantified by ImageJ of $(C){ }^{* * *} \mathrm{P}<0.001$ vs. control. ns, non-significant; VD, vitamin $\mathrm{D}$; miR, microRNA; Rsbn1, round spermatid basic protein 1.

A

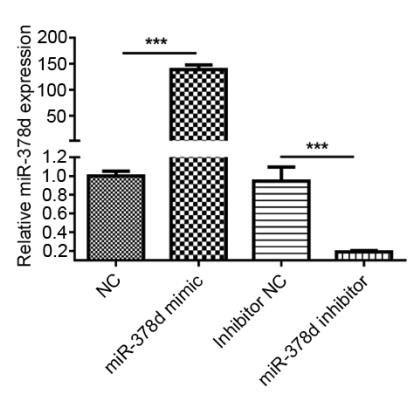

B

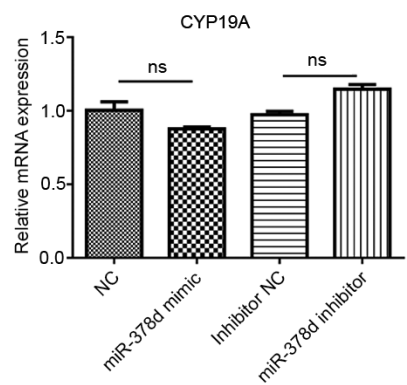

D

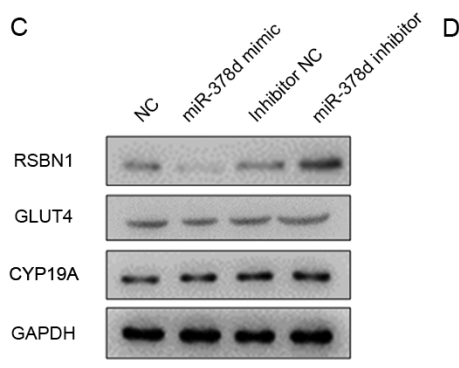

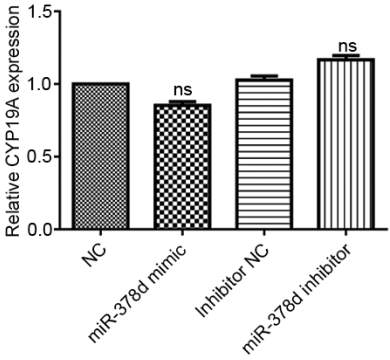
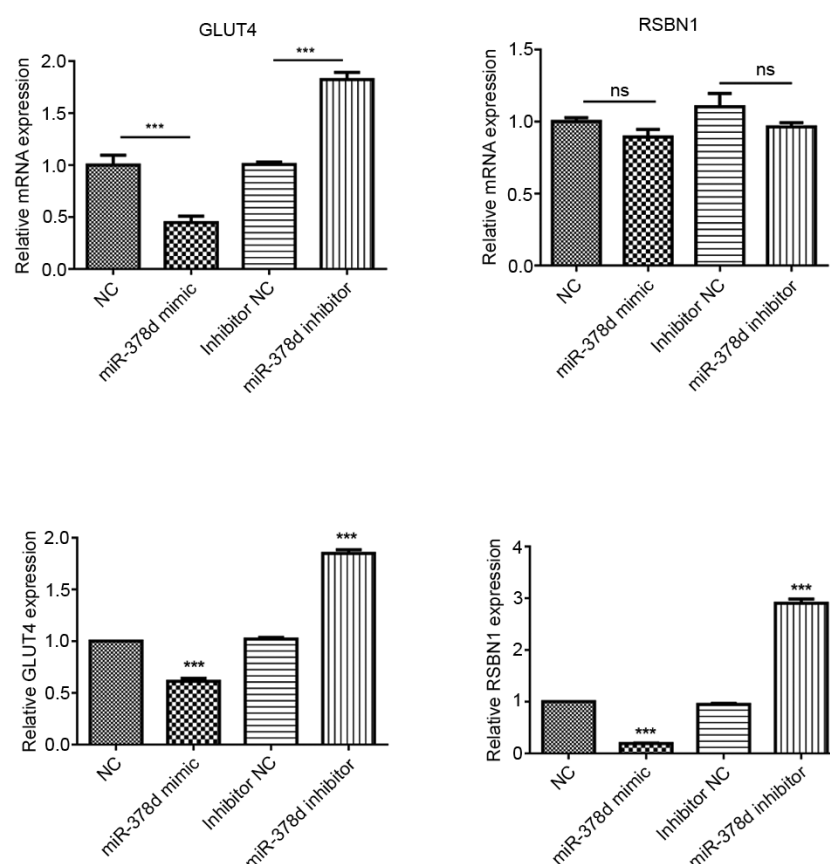

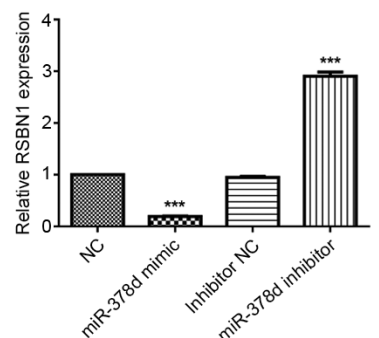

Figure 3. miR-378d post-transcriptionally regulates Rsbn1 in ovaries. (A) Reverse transcription-quantitative PCR results showed that mir-378d was significantly upregulated in the mimic group and significantly downregulated in the inhibitor group. (B) Glut4 mRNA expression was decreased significantly in the mimic group and increased significantly in the inhibitor group, while Rsbn1 and Cyp19a mRNA expression levels showed no significant differences. (C) Western blot analyses of Rsbn1, Glut4 and Cyp19a expression levels in mouse granulosa cells transfected with miR-378d mimics or inhibitors. (D) Expression levels of Rsbnl and Glut4 were decreased after mir-378d overexpression, and the expression levels of Rsbnl and Glut4 were increased after mir-378d inhibition, while Cyp19a showed no significant change. ${ }^{* * *} \mathrm{P}<0.001$ vs. respective NC. ns, non-significant; VD, vitamin D; miR, microRNA; Rsbnl, round spermatid basic protein 1; NC, negative control; Glut4, glucose transporter 4; Cyp19a, aromatase.

Glut4 mRNA expression was significantly decreased in the miR-378d mimic group and significantly increased in the inhibitor group, while Rsbnl and Cyp19a mRNA expression levels showed no significant differences (Fig. 3B). The western blotting results demonstrated that the expression levels of Rsbn1 and Glut4 were decreased after miR-378d 


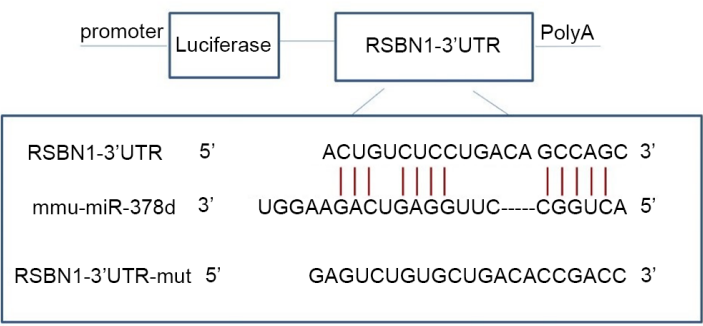

B

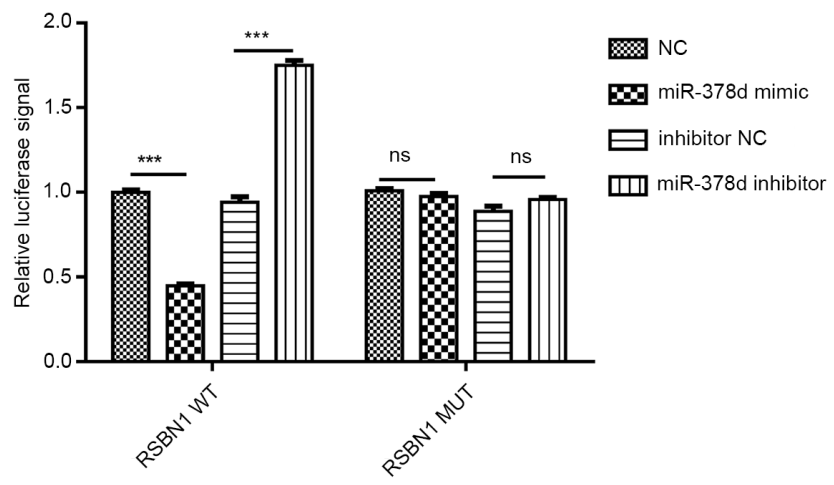

Figure 4. Luciferase reporter gene activity shows that miR-378d directly targets $R s b n 1$ in mouse granulosa cells. (A) Predicted target site of miR-378d in 3'UTR of Rsbn1 mRNA of mice. (B) A luciferase activity assay was performed in the presence of WT Rsbn1 3'UTR or MUT compared with the control. $(\mathrm{n}=3) .{ }^{* * * *} \mathrm{P}<0.001$. WT, wild-type; MUT, mutant; UTR, untranslated region; miR, microRNA; Rsbnl, round spermatid basic protein 1 ; NC, negative control.

overexpression, but were increased after miR-378d inhibition, while Cyp19a showed no significant change (Fig. 3C and D). These results further verified that miR-378d directly regulates $R S B N 1$ in mouse granulosa cells.

miR-378d targets 3'UTR of RSBN1 mRNA. WT and MUT plasmids of Rsbn1 3'UTR were constructed according to their binding sites. After co-transfection with miR-378d mimic or inhibitor, the results of luciferase reporter gene assays revealed the following: WT Rsbn1 3'UTR activity was decreased by mir-378d mimic $(\mathrm{P}<0.001)$ and increased by miR-378d inhibitor, while miR-378d mimic and inhibitor had no significant effect on MUT 3'UTR. These results indicate that miR-378d can interact with Rsbnl mRNA and bind to Rsbnl mRNA (Fig. 4A and B), thus having a targeting role.

It was then investigated using rescue experiments whether the Rsbnl was a real functional target of miR-378d in mouse granulosa cells. Western blot analysis demonstrated that overexpression of miR-378d could downregulate the expression levels of Rsbnl and Glut4. After overexpression of Rsbnl, the protein expression levels of Rsbnl and Glut4 were significantly upregulated. Moreover, after co-transfection with Rsbnl OE and miR378d mimic, Rsbnl and Glut4 expression levels were not significantly change (Fig. 5A and B). It was found that the downregulation of Rsbnl after the overexpression of miR-378d was reversed by the overexpression of $R s b n 1$, while Cyp19a showed no significant change. Inhibition of miR-378d could upregulate the expression levels of Rsbn1 and Glut4 (Fig. 5C). After knocking down Rsbnl, the protein expression levels of Rsbnl and Glut4 were significantly downregulated. Furthermore, after co-transfection with Rsbnl knockdown and miR378d inhibitor, the protein expression levels of $R s b n 1$ and Glut4 did not significantly change in comparison with NC, which may be because of the upregulating ability of miR-378d on Rsbnl (Fig. 5C). It was identified that Glut4 was decreased by the knockdown of Rsbn1, while Cyp19a expression did not significantly change (Fig. 5C). In addition, RT-qPCR results demonstrated that, compared with the control group, the mRNA expression of Glut4 was significantly decreased after overexpression of miR-378d, while the mRNA expression levels of Rsbnl and Cyp19a did not significantly change (Fig. 5D). After the overexpression of Rsbn1, the mRNA expression levels of Rsbnl and Glut4 were significantly increased, but Cyp19a mRNA expression did not change (Fig. 5D).

\section{Discussion}

Accumulating evidence has suggested that VD is directly or indirectly associated with fertility disorders in women (32). Moreover, VD deficiency is associated with a decrease in calcium and phosphorus. Sun et al (33) reported that when calcium and phosphorus are supplemented to the mice with VDR or 1-a hydroxylase gene knockout; their reproductive dysfunctions could be corrected, suggesting that the effects of $\mathrm{VD}$ on the fertility in female mice is achieved by regulating the levels of calcium and phosphorus. From a genetic point of view, mutations in VD-related genes in diabetic patients may be associated with glucose tolerance and inflammatory responses (34). Aghajafari et al (35) conducted a systematic review and meta-analysis, which revealed that VD deficiency in pregnant women could increase the risk of gestational diabetes, preeclampsia and premature birth. Furthermore, VD supplementation can antagonize the negative effects of preeclampsia on the number and function of fetal endothelial cell colony-forming cells (36). Another study that performed in vitro fertilization and embryo transfer (IVF-ET) for 132 infertile women reported that the $25(\mathrm{OH}) \mathrm{D}$ levels in the serum and follicular fluid of non-pregnant women were significantly lower compared with those of pregnant women. At the same time, implantation rates were also relatively low in patients with low VD levels (37). A different study that determined the VD levels in the serum and follicular fluid of 173 women after IVF-ET showed that there were no significant differences in the gonadotropin dose and endometrial thickness between the two groups of women with adequate or insufficient 25(OH)D3 levels (13). Moreover, Polyzos et al (38) found that VD was associated with pregnancy rates during assisted reproductive technology treatment. During the treatment with ovulation drugs, both the number of formed mature oocytes and pregnancy rates were much higher in the patients with high VD levels compared with those found in patients with low VD levels.

The application of VD-deficient feed combined with a UV-free environment is one of the most commonly used methods to establish animal models with VD deficiency, which is also in line with the current characteristics of reduced VD levels caused by unbalanced diet structure and reduced daylight exposure (39). The mouse model of VD deficiency was established as previously described (28), and transcriptome sequencing 


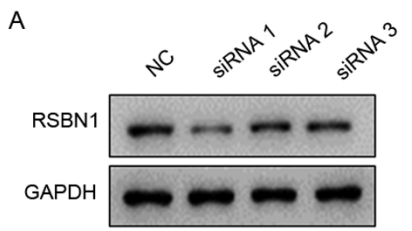

B
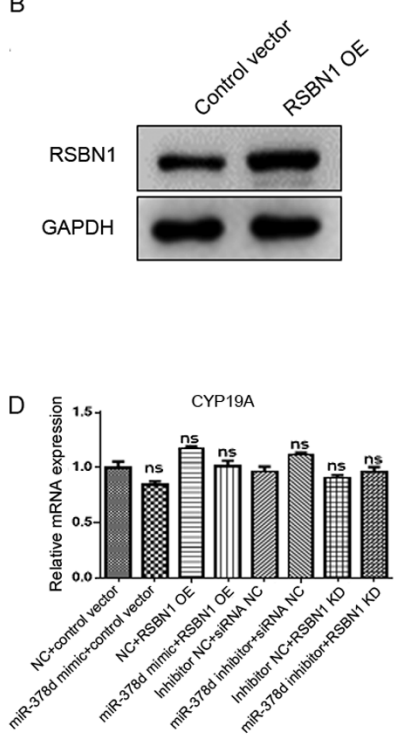
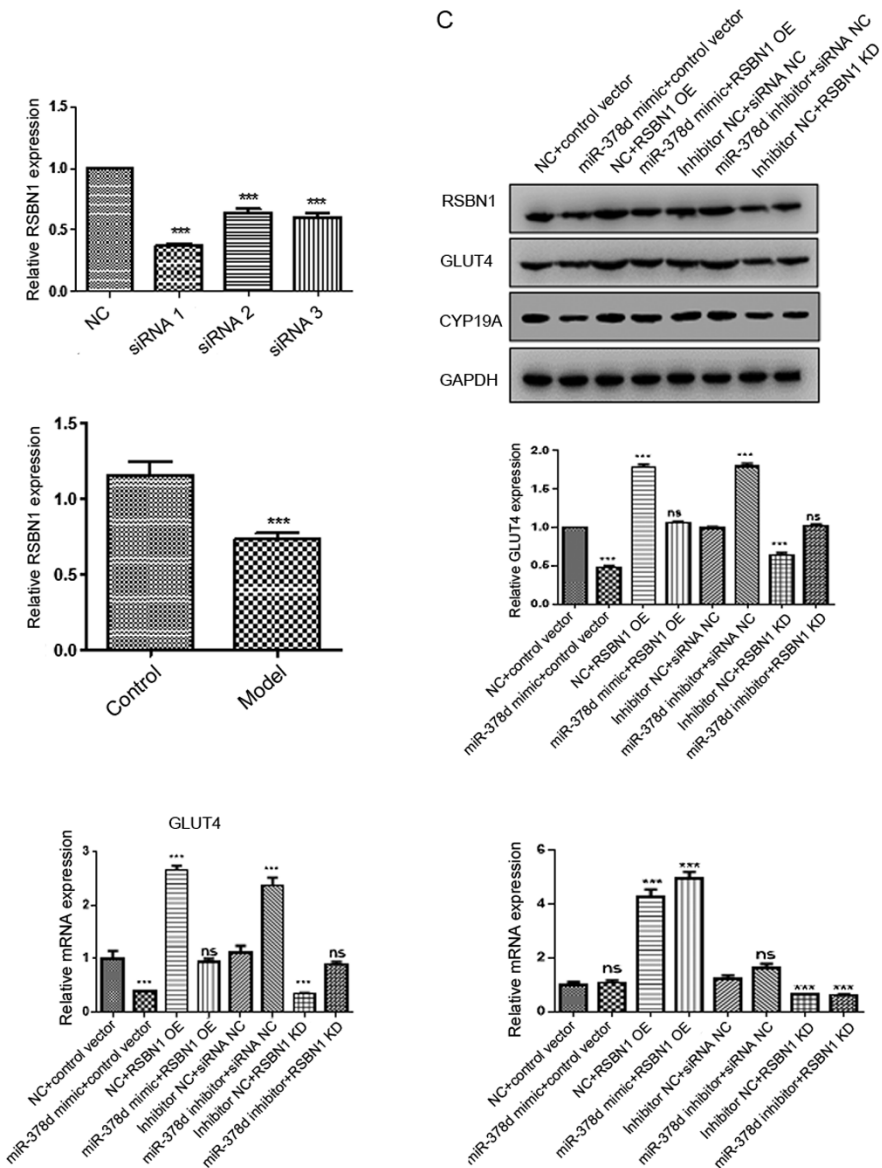
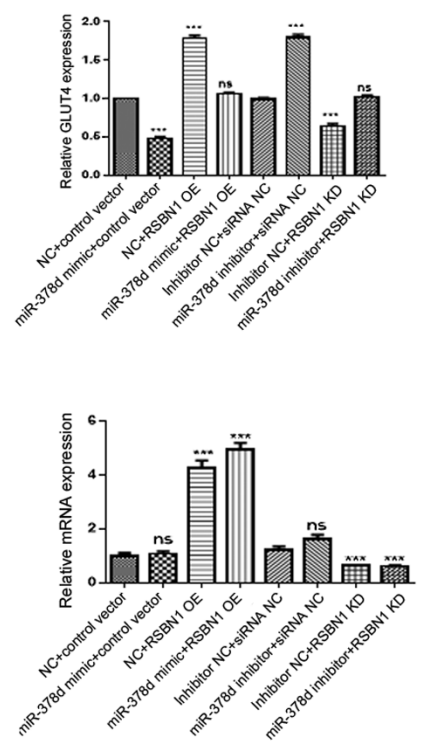
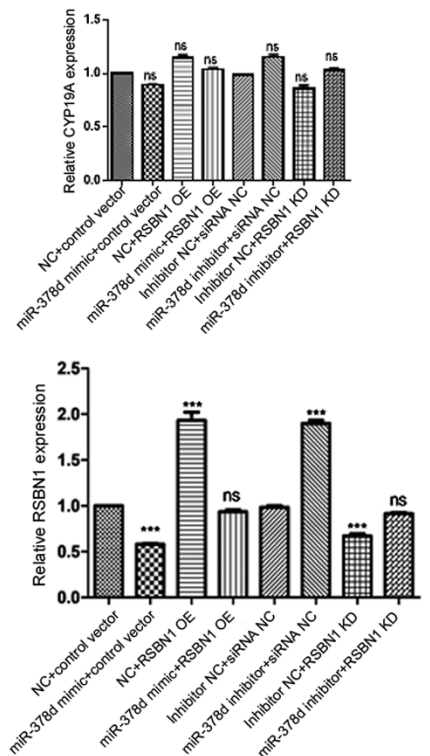

Figure 5. Rsbn1 is a functional target of miR-378d in mouse granulosa cells. (A) Western blotting results showed that siRNA 1 interference was the most efficient. (B) Western blotting results demonstrated that Rsbn1 expression was upregulated in the overexpression group. (C) Expression levels of Rsbn1, Glut4 and Cyp19a were detected via western blotting after overexpression or knockdown of miR-378d. (D) mRNA expression levels of Rsbn1, Glut4 and Cyp19a were detected via reverse transcription-quantitative PCR after miR-378d interference. ${ }^{* * *} \mathrm{P}<0.001$. miR, microRNA; Rsbn1, round spermatid basic protein 1 ; NC, negative control; Glut4, glucose transporter 4; Cyp19a, aromatase; OE, overexpression; siRNA, small interfering RNA; ns, non-significant.

analysis revealed that miR-378d was abnormally overexpressed in the ovarian tissues of VD-deficient mice, which was further confirmed using RT-qPCR of the extracted ovarian tissues from the mice. At present, there are few studies on the mechanisms of miR-378d, and these mainly focus on the analysis of expression differences $(40,41)$. Kurowska et al (42) have reported that the downregulation of miR-378d may be used as a molecular marker of arthritis progression. Moreover, spectrum analysis of miRNA differences using a microarray platform revealed that the expression of miR-378d was significantly higher in colitis mucosa compared with in uninflamed mucosa (43).

The essential aspect of studying the biological functions and mechanisms of miRNAs is the accurate identification of the target genes of the miRNAs (44). In order to further investigate the molecular mechanisms of miR-378d in the ovaries of the VD-deficient mice, bioinformatics software was used in the current to perform prediction analysis, which revealed that $R s b n 1$ may be the target gene of miR-378d.

Rsbnl is a potential target gene of HIF-1 $\alpha$, which may be involved in a variety of pathophysiological processes. Ohyama et al (45) reported that the expression of Rsbnl in mature testis is a sign of spermatogenesis since its expression increases during the development of round spermatids in male mice. $R s b n l$ is also associated with the expression of anti-Mullerian hormone (AMH), as well as is closely associated with the production of steroid hormones $(45,46)$. Herein, it was found that, compared with the control group, the protein expression levels of Rsbnl in the ovaries of VD-deficient mice were significantly decreased, while there were no significant changes in the mRNA expression levels. Furthermore, the double reporter gene experiments confirmed that miR-378d could bind with Rsbnl mRNA to exert its targeting role. Functional rescue experiments demonstrated that overexpression of miR-378d could downregulate the expression levels of Rsbnl and Glut4 proteins. Moreover, knocking down Rsbnl could downregulate the protein expression levels of Rsbnl and Glut4, while simultaneously inhibiting miR-378d and knocking down Rsbnl had no significant effect on the protein expression levels of Rsbnl and Glut4. These results suggested that in the absence of VD, miR-378d was abnormally increased, and the expression of $R s b n l$ protein was downregulated, thus affecting the expression of downstream molecule Glut4, which may lead to insulin resistance. Regardless of overexpression or knockdown of miR-378d, Cyp19a showed no obvious changes, therefore suggesting that miR-378d may not be significantly associated with the production of steroid hormones. This finding is not in line with a previous study reporting that miR-378d could regulate the production of estradiol in pig ovaries by targeting the aromatase (47). At the same time, it suggests that there is no clear correlation between 
VD and AMH, which is consistent with the conclusions of Lata et al (48) and Pearce et al (49).

In summary, the present study demonstrated that miR-378d was abnormally upregulated in the ovaries of VD deficient mice, which could negatively regulate $R s b n 1$, eventually affecting the expression of its downstream molecule Glut4. Furthermore, this study revealed a potential molecular marker for the mechanistic studies of VD deficiency, as well as provided novel insights for the diagnosis and treatment of insulin resistance.

\section{Acknowledgements}

Not applicable.

\section{Funding}

The present study was supported by the Changezhou Sci \& Tech Program (grant no. CJ20200103), Changezhou Sci \& Tech Program (grant no.CJ20180028), the Young Scientists Foundation of Changzhou No. 2 People's Hospital (grant no. 2019K009) and the Guiding Science and Technology Project of Changzhou Municipal Health Commission (grant no. WZ201815).

\section{Availability of data and materials}

The datasets used and/or analyzed during the present study are available from the corresponding author on reasonable request.

\section{Authors' contributions}

HS and FX conducted the studies, participated in collecting data and drafted the manuscript. HS, YShang and XC are responsible for confirming the authenticity of the raw data. YShi, HS and FX performed the statistical analysis and participated in its design. HS, YShang and XC participated in acquisition, analysis and interpretation of data, and draft the manuscript. All authors read and approved the final manuscript.

\section{Ethics approval and consent to participate}

All procedures related to animal use were approved by the Animal Care and Use Committee of Nanjing Medical University. This study was carried out in strict accordance with the recommendations in the Guide for the Care and Use of Laboratory Animals of the National Institutes of Health.

\section{Patient consent for publication}

Not applicable.

\section{Competing interests}

The authors declare that they have no competing interests

\section{References}

1. Holick MF: Vitamin D deficiency. N Engl J Med 357: 266-281, 2007

2. Holick MF, Binkley NC, Bischoff-Ferrari HA, Gordon CM, Hanley DA, Heaney RP, Murad MH and Weaver CM: Guidelines for preventing and treating vitamin D deficiency and insufficiency revisited. J Clin Endocrinol Metab 97: 1153-1158, 2012.
3. Nerhus M, Berg AO, Simonsen C, Haram M, Haatveit B, Dahl SR, Gurholt TP, Bjella TD, Ueland T, Andreassen OA, et al: Vitamin D Deficiency Associated With Cognitive Functioning in Psychotic Disorders. J Clin Psychiatry 78: e750-e757, 2017.

4. Ponsonby AL, McMichael A and van der Mei I: Ultraviolet radiation and autoimmune disease: Insights from epidemiological research. Toxicology 181-182: 71-78, 2002.

5. Ford JA, MacLennan GS, Avenell A, Bolland M, Grey A and Witham M; RECORD Trial Group: Cardiovascular disease and vitamin D supplementation: Trial analysis, systematic review, and meta-analysis. Am J Clin Nutr 100: 746-755, 2014.

6. Gagnon C, Lu ZX, Magliano DJ, Dunstan DW, Shaw JE, Zimmet PZ, Sikaris K, Ebeling PR and Daly RM: Low serum 25-hydroxyvitamin $\mathrm{D}$ is associated with increased risk of the development of the metabolic syndrome at five years: results from a national, population-based prospective study (The Australian Diabetes, Obesity and Lifestyle Study: AusDiab). J Clin Endocrinol Metab 97: 1953-1961, 2012.

7. Muscogiuri G, Mitri J, Mathieu C, Badenhoop K, Tamer G, Orio F, Mezza T, Vieth R, Colao A and Pittas A: Mechanisms in endocrinology: Vitamin D as a potential contributor in endocrine health and disease. Eur J Endocrinol 171: R101-R110, 2014.

8. van Schoor N and Lips P: Global Overview of Vitamin D Status. Endocrinol Metab Clin North Am 46: 845-870, 2017.

9. Tanamura A, Nomura S, Kurauchi O, Furui T, Mizutani S and Tomoda Y: Purification and characterization of 1,25(OH)2D3 receptor from human placenta. J Obstet Gynaecol (Tokyo 1995) 21: 631-639, 1995.

10. Pérez-Fernandez R, Alonso M, Segura C, Muñoz I, García-Caballero $\mathrm{T}$ and Diguez C: Vitamin $\mathrm{D}$ receptor gene expression in human pituitary gland. Life Sci 60: 35-42, 1997.

11. Thill M, Becker S, Fischer D, Cordes T, Hornemann A, Diedrich K, Salehin D and Friedrich M: Expression of prostaglandin metabolising enzymes COX-2 and 15-PGDH and VDR in human granulosa cells. Anticancer Res 29: 3611-3618, 2009.

12. Parikh G, Varadinova M, Suwandhi P, Araki T, Rosenwaks Z, Poretsky L and Seto-Young D: Vitamin D regulates steroidogenesis and insulin-like growth factor binding protein-1 (IGFBP-1) production in human ovarian cells. Horm Metab Res 42: 754-757, 2010.

13. Zhao J, Huang X, Xu B, Yan Y, Zhang Q and Li Y: Whether vitamin D was associated with clinical outcome after IVF/ICSI: A systematic review and meta-analysis. Reprod Biol Endocrinol 16: 13, 2018.

14. Jiang R, Ding L, Zhou J, Huang C, Zhang Q, Jiang Y, Liu J, Yan Q, Zhen X, Sun J, et al: Enhanced HOXA10 sumoylation inhibits embryo implantation in women with recurrent implantation failure. Cell Death Discov 3: 17057, 2017.

15. Wei Z, Yoshihara E, He N, Hah N, Fan W, Pinto AFM, Huddy T, Wang Y, Ross B, Estepa G, et al: Vitamin D Switches BAF Complexes to Protect $\beta$ Cells. Cell 173: 1135-1149.e15, 2018.

16. Liang Y, Ridzon D, Wong L and Chen C: Characterization of microRNA expression profiles in normal human tissues. BMC Genomics 8: 166, 2007.

17. Jiang XP, Ai WB, Wan LY, Zhang YQ and Wu JF: The roles of microRNA families in hepatic fibrosis. Cell Biosci 7: 34, 2017.

18. Weng WH, Leung WH, Pang YJ and Hsu HH: Lauric acid can improve the sensitization of Cetuximab in KRAS/BRAF mutated colorectal cancer cells by retrievable microRNA-378 expression. Oncol Rep 35: 107-116, 2016.

19. Ji KX, Cui F, Qu D, Sun RY, Sun P, Chen FY, Wang SL and Sun HS: miR-378 promotes the cell proliferation of non-small cell lung cancer by inhibiting FOXG1. Eur Rev Med Pharmacol Sci 22: 1011-1019, 2018

20. Ganesan J, Ramanujam D, Sassi Y, Ahles A, Jentzsch C, Werfel S, Leierseder S, Loyer X, Giacca M, Zentilin L, et al: miR-378 controls cardiac hypertrophy by combined repression of mitogen-activated protein kinase pathway factors. Circulation 127: 2097-2106, 2013.

21. Hyun J, Wang S, Kim J, Rao KM, Park SY, Chung I, Ha CS, Kim SW, Yun YH and Jung Y: MicroRNA-378 limits activation of hepatic stellate cells and liver fibrosis by suppressing Gli3 expression. Nat Commun 7: 10993, 2016.

22. Ishida M, Shimabukuro M, Yagi S, Nishimoto S, Kozuka C, Fukuda D, Soeki T, Masuzaki H, Tsutsui M and Sata M: MicroRNA-378 regulates adiponectin expression in adipose tissue: A new plausible mechanism. PLoS One 9: e111537, 2014.

23. Gungormez C, Gumushan Aktas H, Dilsiz N and Borazan E: Novel miRNAs as potential biomarkers in stage II colon cancer: Microarray analysis. Mol Biol Rep 46: 4175-4183, 2019. 
24. Meerson A, Eliraz Y, Yehuda H, Knight B, Crundwell M, Ferguson D, Lee BP and Harries LW: Obesity impacts the regulation of miR-10b and its targets in primary breast tumors. BMC Cancer 19: 86, 2019.

25. Dupont J and Scaramuzzi RJ: Insulin signalling and glucose transport in the ovary and ovarian function during the ovarian cycle. Biochem J 473: 1483-1501, 2016.

26. Laganà AS, Vitale SG, Ban Frangež H, Vrtačnik-Bokal E and D'Anna R: Vitamin D in human reproduction: The more, the better? An evidence-based critical appraisal. Eur Rev Med Pharmacol Sci 21: 4243-4251, 2017.

27. Zarnani AH, Shahbazi M, Salek-Moghaddam A, Zareie M, Tavakoli M, Ghasemi J, Rezania S, Moravej A, Torkabadi E, Rabbani H, et al: Vitamin D3 receptor is expressed in the endometrium of cycling mice throughout the estrous cycle. Fertil Steril 93: 2738-2743, 2010

28. Li P, Xu X, Cao E, Yu B, Li W, Fan M, Huang M, Shi L, Zeng R, $\mathrm{Su} \mathrm{X}$, et al: Vitamin D deficiency causes defective resistance to Aspergillus fumigatus in mice via aggravated and sustained inflammation. PLoS One 9: e99805, 2014.

29. Lamas S, Carvalheira J, Gartner F and Amorim I: C57BL/6J mouse superovulation: Schedule and age optimization to increase oocyte yield and reduce animal use. Zygote: Jan 15, 2021 (Epub ahead of print). doi: 10.1017/S0967199420000714

30. Livak KJ and Schmittgen TD: Analysis of relative gene expression data using real-time quantitative PCR and the 2(-Delta Delta C(T)) Method. Methods 25: 402-408, 2001.

31. Mao Z, Zhao H, Qin Y, Wei J, Sun J, Zhang W and Kang Y: Post-Transcriptional Dysregulation of microRNA and Alternative Polyadenylation in Colorectal Cancer. Front Genet 11: 64, 2020.

32. Lerchbaum E and Obermayer-Pietsch B: Vitamin D and fertility: A systematic review. Eur J Endocrinol 166: 765-778, 2012.

33. Sun W, Xie H, Ji J, Zhou X, Goltzman D and Miao D: Defective female reproductive function in $1,25(\mathrm{OH}) 2 \mathrm{D}$-deficient mice results from indirect effect mediated by extracellular calcium and/or phosphorus. Am J Physiol Endocrinol Metab 299: E928-E935, 2010.

34. Takiishi T, Gysemans C, Bouillon R and Mathieu C: Vitamin D and diabetes. Rheum Dis Clin North Am 38: 179-206, 2012.

35. Aghajafari F, Nagulesapillai T, Ronksley PE, Tough SC, O'Beirne M and Rabi DM: Association between maternal serum 25-hydroxyvitamin D level and pregnancy and neonatal outcomes: Systematic review and meta-analysis of observational studies. BMJ 346 (mar26 4): f1169, 2013.

36. von Versen-Höynck F, Brodowski L, Dechend R, Myerski AC and Hubel CA: Vitamin D antagonizes negative effects of preeclampsia on fetal endothelial colony forming cell number and function. PLoS One 9: e98990, 2014.

37. Fung JL, Hartman TJ, Schleicher RL and Goldman MB: Association of vitamin D intake and serum levels with fertility: Results from the Lifestyle and Fertility Study. Fertil Steril 108: 302-311, 2017.

38. Polyzos NP, Anckaert E, Guzman L, Schiettecatte J, Van Landuyt L, Camus M, Smitz J and Tournaye H: Vitamin D deficiency and pregnancy rates in women undergoing single embryo, blastocyst stage, transfer (SET) for IVF/ICSI. Hum Reprod 29: 2032-2040, 2014.
39. Duchow EG, Cooke NE, Seeman J, Plum LA and DeLuca HF: Vitamin D binding protein is required to utilize skin-generated vitamin D. Proc Natl Acad Sci USA 116: 24527-24532, 2019.

40. Liu C, Lai Y, Ying S, Zhan J and Shen Y: Plasma exosome-derived microRNAs expression profiling and bioinformatics analysis under cross-talk between increased low-density lipoprotein cholesterol level and ATP-sensitive potassium channels variant rs1799858. J Transl Med 18: 459, 2020.

41. Zhang X, Mens MMJ, Abozaid YJ, Bos D, Darwish Murad S, de Knegt RJ, Ikram MA, Pan Q and Ghanbari M: Circulatory microRNAs as potential biomarkers for fatty liver disease: The Rotterdam study. Aliment Pharmacol Ther 53: 432-442, 2021.

42. Kurowska W, Kuca-Warnawin E, Radzikowska A, Jakubaszek M, Maślińska M,Kwiatkowska B and Maśliński W: Monocyte-related biomarkers of rheumatoid arthritis development in undifferentiated arthritis patients - a pilot study. Reumatologia 56: 10-16, 2018.

43. Valmiki S, Ahuja V and Paul J: MicroRNA exhibit altered expression in the inflamed colonic mucosa of ulcerative colitis patients. World J Gastroenterol 23: 5324-5332, 2017.

44. Bolha L, Pižem J, Frank-Bertoncelj M, Hočevar A, Tomšič M and Jurčić V: Identification of microRNAs and their target gene networks implicated in arterial wall remodelling in giant cell arteritis. Rheumatology (Oxford) 59: 3540-3552, 2020.

45. Ohyama K, Ohta M, Hosaka YZ, Tanabe Y, Ohyama T and Yamano Y: Expression of anti-Müllerian hormone and its type II receptor in germ cells of maturing rat testis. Endocr J 62: 997-1006, 2015.

46. Abu-Jamous B, Buffa FM, Harris AL and Nandi AK: In vitro downregulated hypoxia transcriptome is associated with poor prognosis in breast cancer. Mol Cancer 16: 105, 2017.

47. Xu S,Linher-Melville K, Yang BB, Wu D and Li J: Micro-RNA378 (miR-378) regulates ovarian estradiol production by targeting aromatase. Endocrinology 152: 3941-3951, 2011.

48. Lata I, Tiwari S, Gupta A, Yadav S and Yadav S: To Study the Vitamin D Levels in Infertile Females and Correlation of Vitamin D Deficiency with AMH Levels in Comparison to Fertile Females. J Hum Reprod Sci 10: 86-90, 2017.

49. Pearce K, Gleeson K and Tremellen K: Serum anti-Mullerian hormone production is not correlated with seasonal fluctuations of vitamin D status in ovulatory or PCOS women. Hum Reprod 30: 2171-2177, 2015. International (CC BY-NC-ND 4.0) License. 